\title{
ON NILPOTENCY OF THE SEPARATING IDEAL OF A DERIVATION
}

\author{
RAMESH V. GARIMELLA
}

(Communicated by Palle E. T. Jorgensen)

\begin{abstract}
We prove that the separating ideal $S(D)$ of any derivation $D$ on a commutative unital algebra $B$ is nilpotent if and only if $S(D) \cap\left(\cap R^{n}\right)$ is a nil ideal, where $R$ is the Jacobson radical of $B$. Also we show that any derivation $D$ on a commutative unital semiprime Banach algebra $B$ is continuous if and only if $\bigcap(S(D))^{n}=\{0\}$. Further we show that the set of all nilpotent elements of $S(D)$ is equal to $\bigcap(S(D) \cap P)$, where the intersection runs over all nonclosed prime ideals of $B$ not containing $S(D)$. As a consequence, we show that if a commutative unital Banach algebra has only countably many nonclosed prime ideals then the separating ideal of a derivation is nilpotent.
\end{abstract}

\section{INTRODUCTION}

In [9] Singer and Wermer proved that the range of a continuous derivation on a commutative Banach algebra is contained in the Jacobson radical. In the same paper they conjectured that the assumption of continuity is not necessary. In [10] Thomas proved the Singer-Wermer conjecture. Still, the answers to the following problems seem to be open.

(Q1) Is the separating ideal of a derivation on a commutative unital Banach algebra nilpotent?

(Q2) Are derivations continuous on a commutative unital semiprime Banach algebra?

(Q3) Are derivations continuous on integral domains?

It is straightforward to notice that the above questions are equivalent (see 
$P$ of $B$ not containing $S(D)$. As a corollary, we prove that $S(D)$ is nilpotent if the Banach algebra has only countably many nonclosed prime ideals.

Throughout the following we suppose that $B$ is a commutative unital Banach algebra. $R$ and $N$ will denote, respectively, the Jacobson and nil radicals of $B$. $N$ is also called the prime radical of $B$ and consists of all nilpotent elements of $B . N$ is also equal to the intersection of all prime ideals of $B$. Recall that $B$ is said to be an integral domain if $\{0\}$ is a prime ideal. $B$ is said to be semiprime if it has no nonzero nilpotent elements. For any derivation $D$ on a commutative unital Banach algebra $B$, let $S(D)=\{x \in B$ : there is $x_{n} \rightarrow 0$ with $\left.D x_{n} \rightarrow x\right\}$ be the separating ideal of $B$. It is easy to see that $S(D)$ is a closed ideal of $B$, and by the closed graph theorem it follows that $D$ is continuous on $B$ if and only if $S(D)=\{0\}$. For any ideal $I$, let

$$
(I: D)=\left\{x \in I: D^{n}(x) \in I \text { for each } n \geq 1\right\} .
$$

For any prime ideal $P$ of $B$ it is easy to verify that $(P: D)$ is also a prime ideal. An ideal $I$ of $B$ is said to be nil if each element of $I$ is nilpotent. If $I$ is an ideal of $B$, let $I^{n}$ denote the ideal of $B$ that is the linear span of $n$-fold products of elements of $I$. An ideal $I$ of $B$ is said to be nilpotent if $I^{n}=\{0\}$ for some positive integer $n$. It is known that every closed nil ideal is nilpotent [6].

\section{Preliminaries}

Lemma 2.1. Suppose $B$ is a commutative Banach algebra that is also an integral domain. Further suppose that there exists a sequence of nonzero ideals $\left\{I_{n}, n \geq\right.$ 1) such that $\bigcap_{n=1}^{\infty} I_{n}=\{0\}$. Then every derivation on $B$ is continuous.

Proof. Suppose the result is false. Since $B$ is an integral domain, by Remark 3.1 of [5], we may assume that there exists a discontinuous derivation $D$ such that $S(D)$ is the smallest nonzero closed ideal of $B$. Let $\left\{I_{n}, n \geq 1\right\}$ be a sequence of nonzero ideals in $B$ such that $\bigcap_{n=1}^{\infty} I_{n}=\{0\}$. Since $B$ is an integral domain, $I_{n} \cap S(D)$ (which contains $I_{n} S(D)$ ) is a nonzero ideal for each $n$. Since $S(D)$ is the smallest closed ideal of $B, I_{n} \cap S(D)$ is dense in $S(D)$ for each $n$. Let $J_{n}=I_{n} \cap S(D)$ for each $n \geq 1$. Obviously, $\bigcap_{n=1}^{\infty} J_{n}=\{0\}$.

For the remainder of the proof, the argument is similar to the proof of Proposition 3.1 of [4]. Let $x$ be a nonzero element in $S(D)$ such that $\|x\|=1$. Since $\overline{x J_{n}}=S(D)$ for each $n \geq 1$, there exists a sequence $\left\{t_{n}\right\}$ (with $0 \neq t_{n} \in J_{n}$ ) such that $\left\|t_{1} x-x\right\|<4^{-1}$ and

$$
\left\|t_{n} x-x\right\|<4^{-n}\left(1+\left\|t_{1}\right\|\right)^{-1} \cdots\left(1+\left\|t_{n-1}\right\|\right)^{-1} \text { for all } n \geq 2
$$

Put, for each $k \geq 1, n \geq k, c_{k, n}=t_{k} \cdots t_{n} x$. Then for each $k \geq 1, n \geq k$,

$$
\left\|c_{k, n}-c_{k+1, n}\right\| \leq\left\|t_{k} \cdots t_{n}\right\|\left\|x-x t_{n+1}\right\|<4^{-n-1}
$$

So the sequence is Cauchy and hence convergent. Let $s_{k}=\lim _{n \rightarrow \infty} c_{k, n}$. Since $c_{k, n}=t_{k} c_{k+1, n}$ for $n \geq k+1$, we have $s_{k}=t_{k} s_{k+1}$. Hence $s_{1} \in \bigcap_{n=1}^{\infty} J_{n}$. Since $\bigcap_{n=1}^{\infty} J_{n}=\{0\}$, it follows that $s_{1}=0$. 
Since $\sum_{k=1}^{\infty}\left(c_{k+1,1}-c_{k, 1}\right)+c_{1,1}=0$, we have

$$
\begin{aligned}
\|x\| & =\|-x\|=\left\|c_{1,1}+\sum_{k=1}^{\infty}\left(c_{k+1,1}-c_{k, 1}\right)-x\right\| \\
& \leq\left\|c_{1,1}-x\right\|+\sum_{k=1}^{\infty}\left\|c_{k+1,1}-c_{k, 1}\right\| \\
& <4^{-1}+\sum_{k=1}^{\infty} 4^{-k-1}<1 .
\end{aligned}
$$

This is a contradiction to the fact that $\|x\|=1$. Q.E.D.

Lemma 2.2. Let $D$ be a derivation on a commutative unital Banach algebra $B$ such that the separating ideal $S(D)$ of $D$ is not nilpotent. Then there exists a closed prime ideal $P$ satisfying

(i) $P=(P: D)$, i.e., $P$ is invariant under $D$.

(ii) $P$ does not contain either the Jacobson radical $R$ or $S(D)$.

Remark 2.3. By Thomas's theorem [10], since $D(B)$ is contained in $R$, it clearly follows that $S(D)$ is contained in $R$. In the following proof of the above lemma we offer arguments without invoking such a powerful result.

Proof of Lemma 2.2. Since $S(D)$ is not nilpotent by Theorem 2.5 of [1], there exists finitely many minimal prime ideals not containing $S(D)$ such that $S(D) \cap$ $N=P_{1} \cap P_{2} \cap \cdots \cap P_{k} \cap S(D)$, where $N$ is the nil radical of $B$. Suppose $R$ is contained in $P_{i}$ for each $i$. Then $R \cap S(D)$ is contained in $N$. Since $R \cap S(D)$ is a closed ideal, $R \cap S(D)$ is a nilpotent ideal (see [6]). Hence by Lemma 2.1 of [5], $S(D)$ is a nilpotent ideal. This is false. Hence there exists a minimal prime ideal say $P$, which does not contain either $R$ or $S(D)$. Since $(P: D)$ is also a prime ideal contained in $P$ and $P$ being minimal, it follows that $P=(P: D)$. This completes the proof of the lemma.

Proposition 2.4. The following statements are equivalent:

(i) The separating ideal of a derivation on a commutative unital Banach algebra is always nilpotent.

(ii) Derivations are continuous on commutative unital semiprime Banach algebras.

(iii) Derivations are continuous on commutative unital Banach algebras that are integral domains.

Proof. For the equivalence of (ii) and (iii) refer to Theorem 3.1 of [5]. Obviously (i) implies (ii). Suppose (ii) is true and (i) is false. Let $B$ be a commutative Banach algebra and $D: B \rightarrow B$ be a derivation such that $S(D)$ is not nilpotent. Then by Lemma 2.2 there is a closed prime ideal $P$ invariant under $D$ and not containing $S(D)$. Hence $\delta: B / P \rightarrow B / P$, defined by $\delta(x+P)=D(x)+P$, is a well-defined derivation. Since $P$ is a closed prime ideal, $B / P$ is an integral domain. Since every integral domain is semiprime, by our supposition $\delta$ is continuous on $B / P$. That is, the separating ideal $S(\delta)$ of 
$\delta$ is the zero ideal in $B / P$. This implies that $S(D)$ is contained in $P$, which is a contradiction. This completes the proof of the proposition.

\section{MAin Results}

Thomas's theorem [10] implies that the separating ideal of a derivation is contained in the Jacobson radical of the algebra. Hence, if the Jacobson radical is nilpotent then so is the separating ideal of a derivation on the algebra. In [2] Dales constructed a Banach algebra with nilpotent Jacobson radical and a discontinuous derivation using a divisible submodule that is contained in the Jacobson radical (especially, refer to [2, Lemmas 1 and 5 and proof of Theorem 2 in $\S 3]$ ). Also the known examples of discontinuous derivations that map the algebra into a nil divisible ideal contained in $\bigcap_{n=1}^{\infty} R^{n}$, where $R$ is the Jacobson radical of the algebra, have nilpotent separating ideals (refer to [8, $\S 8]$; in particular, Example 8.8, Theorem 8.9, and Remark 8.9). This is not a coincidence as we show in the following that the separating ideal of a derivation is nilpotent if it intersects $\bigcap_{n=1}^{\infty} R^{n}$ in a nil ideal.

Theorem 3.1. Let $R$ be the Jacobson radical of the commutative unital Banach algebra $B$. Let $D$ be any derivation on $B$. Then the separating ideal $S(D)$ is nilpotent if and only if $\left(S(D) \cap\left(\bigcap_{n=1}^{\infty} R^{n}\right)\right)$ is a nil ideal.

Proof. Let $N$ be the nil radical of $B$. One way implication is obvious, so suppose that $\left(S(D) \cap\left(\bigcap_{n=1}^{\infty} R^{n}\right)\right)$ is a nil ideal and $S(D)$ is not nilpotent. By Cusack's theorem [1, Theorem 2.5] there are finitely many closed prime ideals $P_{1}, P_{2}, \ldots, P_{k}$ of $B$ that are minimal and do not contain $S(D)$ such that

$$
S(D) \cap N=S(D) \cap P_{1} \cap P_{2} \cap \cdots \cap P_{k} .
$$

Since each $P_{i}$ is closed, $S(D) \cap N$ is closed. Let $x$ be an element of $S(D)$ that is not nilpotent. Standard automatic continuity theory shows that there is a positive integer $m=m(x)$ such that for each $n \geq m, \overline{x^{n} S(D)}=\overline{x^{m} S(D)}$. The Mittag-Leffler theorem implies that $\bigcap_{n=1}^{\infty} x^{n} S(D)$ is dense in $\overline{x^{m} S(D)}$. It is also clear that $x^{n} S(D) \subseteq R^{n}$ and that $x^{n} S(D) \subseteq S(D)$ for each $n \geq 1$. Therefore we see that

$$
\bigcap_{n=1}^{\infty} x^{n} S(D) \subseteq\left(S(D) \cap\left(\bigcap_{n=1}^{\infty} R^{n}\right)\right) \subseteq S(D) \cap N .
$$

Since $\bigcap_{n=1}^{\infty} x^{n} S(D)$ is dense in $\overline{x^{m} S(D)}$ and $S(D) \cap N$ is closed, it follows that $x^{m} S(D) \subseteq S(D) \cap N$. Therefore we see that $x^{m} S(D) \subseteq P_{i}$ for $i=1,2, \ldots, k$. But each $P_{i}$ is a prime ideal not containing $S(D)$. This forces $x$ to be in each $P_{i}$ and, therefore, $x \in S(D) \cap N$. This implies $x$ is nilpotent, which is a contradiction. Q.E.D.

Theorem 3.2. Let $D$ be a derivation on a commutative unital semiprime Banach algebra $B$. Then $D$ is continuous if and only if $\bigcap_{n=1}^{\infty}(S(D))^{n}=\{0\}$.

Proof. One implication is obvious, so suppose that $\bigcap_{n=1}^{\infty}(S(D))^{n}=\{0\}$. Let $x$ be any element of $S(D)$. Standard automatic continuity theory again shows 
that there is positive integer $m=m(x)$ such that for each $n \geq m, \overline{x^{n} S(D)}=$ $\overline{x^{m} S(D)}$. The Mittag-Leffler theorem implies that $\bigcap_{n=1}^{\infty} x^{n} S(D)$ is dense in $\overline{x^{m} S(D)}$. Since $x^{n} S(D) \subseteq(S(D))^{n+1}$ for each $n \geq 1$, we see that

$$
\bigcap_{n=1}^{\infty} x^{n} S(D) \subseteq \bigcap_{n=1}^{\infty}(S(D))^{n+1}=\{0\}
$$

The only valid conclusion is that $\overline{x^{m} S(D)}=\{0\}$ and, in particular, $x^{m+1}=0$. Since $B$ is assumed to be semiprime, this forces $x=0$. Since $x$ was arbitrary in $S(D)$, we have shown that $S(D)=\{0\}$, and this ends the proof of the theorem.

As mentioned in the proof of Lemma 2.2 and elsewhere, in [1] Cusack showed that for any derivation $D$ on $B$, there exists finitely many minimal prime ideals, say $P_{1}, P_{2}, \ldots, P_{k}$ not containing $S(D)$ such that $S(D) \cap N=S(D) \cap P_{1} \cap \cdots \cap$ $P_{k}$, where $N$ is the nil radical of $B$. Also in the same paper it is noted that all these $P_{i}$ 's are closed. In the following, we prove a similar result involving nonclosed prime ideals of $B$.

Theorem 3.3. Let $S(D)$ be the separating ideal of a derivation $D$ on $B$. Let $N$ be the nil radical of $B$. Then $S(D) \cap N=\bigcap(S(D) \cap P)$, where the intersection on the right-hand side of the equality runs over all nonclosed prime ideals $P$ of $B$ not containing $S(D)$.

Proof. Let $J=\bigcap(S(D) \cap P)$, where the intersection is taken over all nonclosed prime ideals $P$ of $B$. Since $N$ is the intersection of all prime ideals of $B$, obviously $S(D) \cap N$ is contained in $J$. Suppose $S(D) \cap N \neq J$. Let $x$ be a nonzero element of $J$ that is not nilpotent. Since $S(D)$ is contained in the Jacobson radical $R$ (by Thomas's theorem [10]) $x \in R$. By [7, Theorem 2.2, p. 378], there exists a prime ideal $Q$ that is maximal in the set of all ideals of $B$ that are disjoint from the set $S=\left\{x^{n}, n \geq 1\right\}$. Since $x$ is in every nonclosed prime ideal of $B,(Q: D)$ is a prime ideal contained in $Q$, and it follows that $(Q: D)$ is a closed prime ideal of $B$.

Claim $1 . \bigcap_{n=1}^{\infty}\left(x^{n}\right)$ is contained in $(Q: D)$, where $\left(x^{n}\right)$ is the principal ideal generated by $x^{n}$.

Proof of Claim 1. Since $\bigcap_{n=1}^{\infty}\left(x^{n}\right)$ is invariant under $D$, if the claim is false then it is not contained in $Q$. Then $Q+\left(x^{n}\right)$ is an ideal that contains $Q$ properly. By our selection of $Q, S \cap\left(Q+\bigcap_{n=1}^{\infty}\left(x^{n}\right)\right)$ is not empty. That is, there is an integer $m \geq 1$ such that $x^{m}$ belongs to $Q+\bigcap_{n=1}^{\infty}\left(x^{n}\right)$. This implies that there is an element $t$ in $B$ such that $\left(x^{m}-t x^{m+1}\right)$ belongs to $Q$. Since $Q$ is a prime ideal not containing $x$, it follows that $(1-t x)$ belongs to $Q$. This is false because $(1-t x)$ is a unit. Hence our claim is established.

If $(Q: D)=\{0\}$ then $B$ is an integral domain. Also by Claim $1, \bigcap_{n=1}^{\infty}\left(x^{n}\right)=$ $\{0\}$. Hence by Lemma 2.1, it follows that $D$ is continuous. Therefore $S(D)=$ $\{0\}$ and the theorem follows immediately. Thus we may assume that $(Q: D)$ is a nonzero ideal. 
Since $(Q: D)$ is a closed ideal invariant under $D, \partial: B /(Q: D)$ $\rightarrow B /(Q: D)$ defined by $\partial(x+(Q: D))=D(x)+(Q: D)$ is a well-defined derivation. Since $(Q: D)$ is a prime ideal, $B /(Q: D)$ is an integral domain.

Let

$$
I_{n}=\left((Q: D)+\left(x^{n}\right)\right) /(Q: D) \text { for each } n \geq 1 .
$$

Since $x$ does not belong to $(Q: D), I_{n}$ is a nonzero ideal in $B /(Q: D)$ for each $n$.

We now show that $\bigcap_{n=1}^{\infty} I_{n}=\{0\}$ in $B /(Q: D)$. Let $t+(Q: D) \in \bigcap_{n=1}^{\infty} I_{n}$. For each $n \geq 1$, there exists a $c_{n} \in B$ and $q_{n} \in(Q: D)$ such that

$$
t=c_{n} x^{n}+q_{n} .
$$

It is enough to show that $t \in(Q: D)$.

Claim 2. $t \in Q$.

Proof of Claim 2. Suppose it is not true. Since $Q$ is properly contained in $Q+(t)$, where $(t)$ is the principal ideal generated by $t$. By the selection of $Q,(Q+(t)) \cap S \neq \varnothing$. That is, there exists a positive integer $m$ such that $x^{m} \in(Q+(t))$. This implies for some $c$ in $B$ and $q$ in $Q$ that $x^{m}=c t+q$. Since $t$ is also equal to $c_{m+1} x^{m+1}+q_{m+1}$, we have

$$
x^{m}=c\left(c_{m+1} x^{m+1}+q_{m+1}\right)+q .
$$

Hence,

$$
x^{m}\left(1-c c_{m+1} x\right)=\left(c q_{m+1}+q\right) \in Q .
$$

Since $Q$ is a prime ideal and $x$ does not belong to $Q$, it follows that $1-c c_{m+1} x$ belongs to $Q$. Since $x$ is in the Jacobson radical, $1-c c_{m+1} x$ is a unit. This is a contradiction. Hence $t \in Q$.

Claim 3. $D^{n}(t) \in Q$ for each $n \geq 1$.

Proof of Claim 3. Fix an integer $n \geq 1$. We show that $D^{n}(t)$ belongs to $Q$. Suppose it is not true. Since $Q+\left(D^{n}(t)\right)$ properly contains $Q$, where $\left(D^{n}(t)\right)$ is the principal ideal generated by $D^{n}(t)$, there exists positive integer $k$ such that $x^{k}$ belongs to $\left(Q+\left(D^{n}(t)\right)\right)$. That is, there is $q$ in $Q$ and $c$ in $B$ such that $x^{k}=q+c D^{n}(t)$. Now select a positive integer $i$ large enough so that $D^{n}\left(c_{i} x^{i}\right)=b x^{k+1}$ for some $b \in B$.

Since by $(*) \quad t=c_{i} x^{i}+q_{i}$, we have

$$
\begin{aligned}
x^{k} & =q+c D^{n}(t)=q+c D^{n}\left(c_{i} x^{i}+q_{i}\right) \\
& =q+c D^{n}\left(c_{i} x^{i}\right)+c D^{n}\left(q_{i}\right)=q+b x^{k+1}+c D^{n}\left(q_{i}\right) .
\end{aligned}
$$

Hence,

$$
x^{k}(1-b x)=q+c D^{n}\left(q_{i}\right) .
$$

Since $q_{i} \in(Q: D)$, it follows that $D^{n}\left(q_{i}\right) \in Q$. Therefore, by the above equation, it follows that $x^{k}(1-b x) \in Q$. Since $x$ is in the Jacobson radical 
of $B,(1-b x)$ is a unit. Since $Q$ is a prime ideal, this implies $x \in Q$, which is false. This completes the proof of the claim.

Therefore it follows from Claims 2 and 3 that $t$ belongs to $(Q: D)$. Hence $\bigcap_{n=1}^{\infty} I_{n}=\{0\}$ in $B /(Q: D)$. Hence by Lemma 2.1 , it follows that $\partial$ is a continuous derivation on $B /(Q: D)$. Hence $S(\partial)$, the separating ideal of $\partial$, is zero in $B /(Q: D)$. This implies $S(D)$ is contained in $(Q: D)$, and hence, in particular, $S(D)$ is contained in $Q$. Since $x \in S(D)$, we get that $x \in Q$. This is a contradiction to the selection of $Q$. Q.E.D.

For any commutative Banach algebra $B$, let $\mathscr{P}^{\prime}=\mathscr{P}^{\prime}(B)$ be the set of all nonclosed prime ideals of $B$. In [5, Theorem 3.2] we showed that if $\mathscr{P}^{\prime}$ is empty for a commutative unital semiprime Banach algebra then every derivation on the algebra is continuous. In the following we show that if the cardinality of $\mathscr{P}^{\prime}$ is countable, then the separating ideal of every derivation on the algebra (not necessarily semiprime) is nilpotent. As a consequence, it follows that if $\mathscr{P} \prime$ is empty then the separating ideal of every derivation on the algebra is nilpotent. An example of nonsemisimple Banach algebra with $\mathscr{P}^{\prime}$ being empty is a power series algebra

$$
K=K\left(\left(\omega_{n}\right)\right)=\left\{a=\sum_{n=0}^{\infty} a_{n} X^{n}:\|a\|=\sum_{n=0}^{\infty}\left|a_{n}\right| \omega_{n}<\infty\right\}
$$

in one indeterminate $X$ with complex coefficients where $\left\{\omega_{n}: n=0,1,2, \ldots\right\}$ is a sequence in $(0, \infty)$ such that $\omega_{0}=1, \omega_{n+m} \leq \omega_{n} \omega_{m}$, and $\lim \left(\omega_{n}\right)^{1 / n}=0$ (refer to [3, p. 145]). In fact, $K$ has a unique maximal ideal (say) $M=$ $\left\{\sum_{n=0}^{\infty} a_{n} X^{n}: a_{0}=0\right\}$. If $\left\{\omega_{n}\right\}$ is chosen properly, then the only prime ideals of $K$ are $\{0\}$ and $M$. Finally, it is interesting to find some nontrivial examples of Banach algebras with cardinality of $\mathscr{P}^{\prime}$ being countable.

Theorem 3.4. Suppose $B$ is a commutative unital Banach algebra with only countably many nonclosed prime ideals. Then the separating ideal of any derivation on $B$ is nilpotent.

Proof. Suppose $S(D)$ is not nilpotent. By Theorem 2.5 of [1] we have $S(D) \cap$ $N=S(D) \cap P_{1} \cap P_{2} \cap \cdots \cap P_{n}$, where $P_{i}$ 's are minimal prime ideals of $B$ not containing $S(D)$ and $N$ is the nil radical of $B$. Also, the $P_{i}$ 's are closed. Let $P=P_{1}$. Since $P$ is a closed ideal invariant under $D, D$ lifts to a derivation $\partial$ of $B / P$. Since $B$ has only countably many nonclosed prime ideals and there is a one-to-one correspondence between the prime ideals of $B$ containing $P$ and the prime ideals of $B / P$, it follows that $B / P$ has only countably many nonclosed prime ideals. Let $\left\{J_{n}, n \geq 1\right\}$ be the sequence of all nonclosed prime ideals of $B / P$. Since $B / P$ is an integral domain, by Theorem 3.3 it follows that $\bigcap_{n=1}^{\infty}\left(S(\partial) \cap J_{n}\right)=\{0\}$ in $B / P$. Hence by Lemma 2.1, it follows that $\partial$ is continuous on $B / P$. This implies that $S(D)$ is contained in $P$. This is a contradiction that completes the proof of the theorem.

Corollary 3.5. If every prime ideal is closed in $B$ then $S(D)$ is nilpotent. 


\section{ACKNOWLEDGMENT}

I am very grateful to the referee for his valuable comments, suggestions in improving some of the proofs and considerably simplifying the proof of Theorem 3.2 and allowing me to use his proof of Theorem 3.1.

\section{REFERENCES}

1. J. Cusack, Automatic continuity and topologically simple radical Banach algebras, J. London Math. Soc. (2) 21 (1977), 493-500.

2. H. G. Dales, The uniqueness of the functional calculus, Proc. London Math. Soc. 3 (1973), 638-648.

3. __ Automatic continuity: A survey, Bull. London Math. Soc. 10 (1978), 129-183.

4. J. Esterle, Elements for a classification of commutative radical Banach algebras, Proc. Long Beach, 1981, Lecture Notes in Math., vol. 975, Springer-Verlag, Berlin and New York, 1983, pp. 4-65.

5. R. Garimella, Continuity of derivations on some semiprime Banach algebras, Proc. Amer. Math. Soc. 99 (1987), 289-292.

6. S. Grabiner, The nilpotency of Banach nil ideals, Proc. Amer. Math. Soc. 21 (1969), 510.

7. T. W. Hungerford, Algebra, 3rd printing, Springer-Verlag, New York, 1984.

8. A. M. Sinclair, Automatic continuity of linear operators, London Math. Soc. Lecture Notes Ser., vol. 21, Cambridge Univ. Press, Cambridge, 1976.

9. I. M. Singer and J. Wermer, Derivatives on commutative normed algebras, Math. Ann. 129 (1955), 260-264.

10. M. P. Thomas, The image of a derivation is contained in the radical, Ann. of Math. (2) 128 (1988), 435-460.

Department of Mathematics and Statistics, Northwest Missouri State University, MARYVILLE, MisSOURI 64468

E-mail address: 0100142@VAXC.Northwest.Missouri.edu 